\title{
Eco-friendly isolation of tannin based natural colorant from coconut coir (Cocos nucifera) for dyeing of bio-mordanted wool fabric
}

\author{
Adeel $\mathbf{S}^{1}$., Kiran $\mathbf{S}^{1}$., ${ }^{*}$ Yousaf M.S ${ }^{1}$, Habib $\mathrm{N}^{2}$., Hassan, $\mathrm{A}^{3}$. and Hassaan, M. ${ }^{2}$ \\ ${ }^{1}$ Department of Applied Chemistry, Government College University, Faisalabad-38000, Pakistan \\ 2Department of Botany, Government College University, Faisalabad-38000, Pakistan \\ ${ }^{3}$ Department of Chemistry, Federal Urdu University of Arts, Science and Technology, Gulshan-e-Iqbal, Karachi, Pakistan \\ Received: 27/04/2020, Accepted: 18/08/2020, Available online: 09/11/2020 \\ *to whom all correspondence should be addressed: e-mail: shumaila.asimch@gmail.com \\ https://doi.org/10.30955/gnj.003336
}

\section{Graphical abstract}

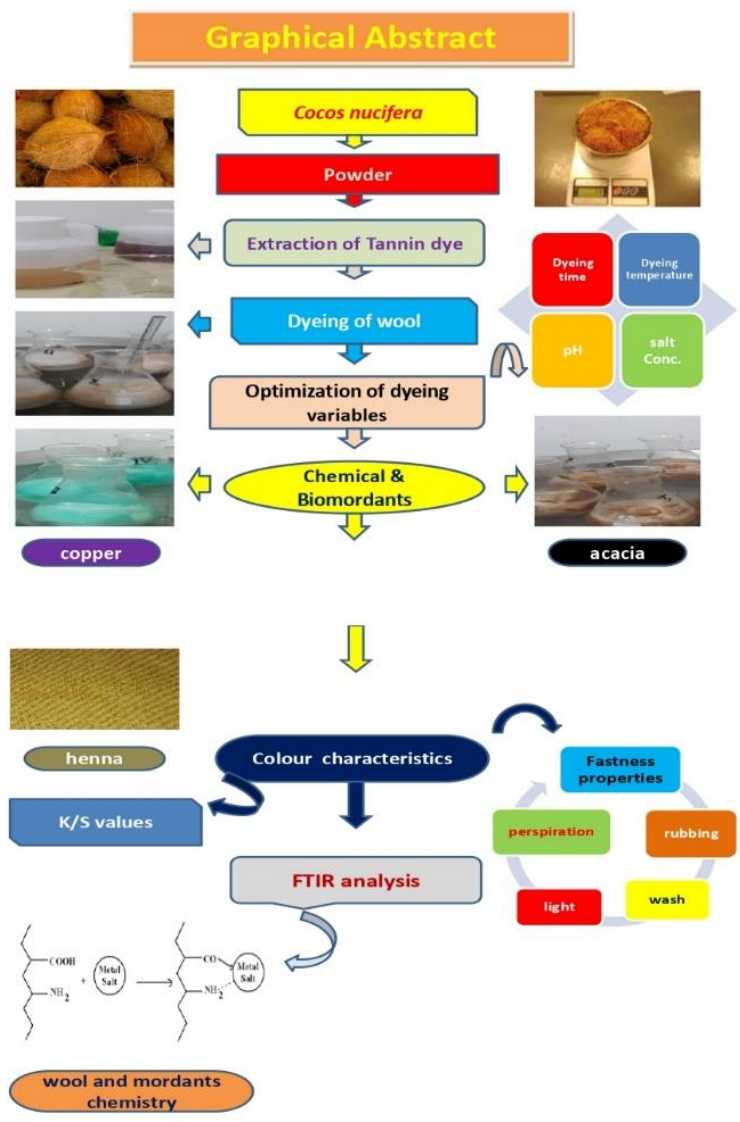

\section{Abstract}

Due to hazardous effluent load of synthetic dyes, the global community is now seeking inclusion of natural products particularly green dyes and pigment in various fields. Current study has been concerned with utilization of eco-friendly tools for isolation of colorants from plant wastes such as coconut coir (Cocos nucifera) for coloration of natural fibers such as wool. Coconut based Tannin was isolated after exposure by microwave treatment for 1-6 min in various media following by optimization of dyeing variables. Excellent yield onto fabric were obtained when coconut coir extract of $1 \mathrm{pH}$ containing $5 \mathrm{~g} / 100 \mathrm{~mL}$ Glauber salt, was used to dye at $65^{\circ} \mathrm{C}$ for $65 \mathrm{~min}$. To develop new shades among mordants used, bio-mordanted wool fabrics exhibited more excellent shades than chemical ones with excellent fastness ratings. It has been concluded that Microwave treatment has excellent potential to isolate natural dye from plant wastes, where the introduction of bio-mordants for shade depth and fastness characteristics has made process more sustainable and viable for natural fibers coloration.

Keywords: Coconut coir, microwave treatment, wool dyeing, chemical mordants, bio-mordants, $\mathrm{K} / \mathrm{S}$ value, colorfastness.

\section{Introduction}

In the present situation, the functional uses of natural dyes are growing globally due to their non-toxic, environment-friendly nature (Haddar et al., 2018) and excellent ayuretic particularly antiviral \& antibacterial as well as anticancer \& antioxidant nature (Baaka et al., 2017). These dyes are obtained from plants, animals \& minerals, having alluring colors which not only attracts human beings but also have gained particular place in textiles, cosmetics, leather etc. (Yusuf et al., 2017). With the existence of man-made dyes, these beautiful colorants went declined in their frequent use, but environmental; health conscious people have shown serious concerns over aftermaths of their effluent load. These serious concerns have led the introduction of these natural dyes once again in all walks of life (Kiumarsi et al., 2017).

Hence, these wonderful benefits have now urged research community to find new source of dyes which possess not only medicinal benefits but also show excellent biological activities. However natural dyes have some issues regarding their extraction and fastness ratings (Samanta, 2020). Various conventional methods are in use for extraction of natural dyes and improvement in their 
behaviour for dyeing of fabrics but these are more time \& energy consuming and not eco-friendly (Shazia et al., 2020). Now these methods have been reshuffled by new radiation techniques which have potential to improve the extraction ability and of colorant dyeing process. Another method for improving fastness is mordanting particularly bio-mordanting methods (Ebrahimi and Gashti, 2015). These techniques include plasma radiation, gamma rays, ultrasonic radiation, ultraviolet radiations (Unal et al., 2020). Among such methods, microwave treatment is more effective for extraction of dyes as well as for surface modification of fabric. This method is less time \& energy consumption, uniform heat distribution, low cost and nontoxic nature (Karadag et al., 2020). Microwave radiations provide smooth heat and increase the movement of ions towards the fabric surface. These rays can easily penetrate in all particles of the material, interact with the surface \& distribute the heat in an instant and uniform way. These rays also tune the surface of fabric physically so that color particles approach the fibers inside the scope of attractive powers (Ebrahimi and Gashti, 2016). Biomordants are found to be more superior to chemical mordants due to the certain toxic effects associated with chemical mordants (Mansour et al., 2016). Biomordants enhance the tone and fastness properties of materials dyed with natural dyes. It has been observed that biomordants intensify the dye exhaustion, shade variation and other fibre properties (Shabbir et al., 2017). In view of these benefits this study has been conducted to explain the potential of coconut coir for wool dyeing (Figure 1).

Wool is the semi-crystalline proteinaceous material that contains polyamide as functional unit to interact with dye (Figure 1b). Wool strands have an amphoteric character because of the nearby acidic carboxyl and hydroxyl groups which form peptide linkage and make firm $\mathrm{H}$-bonding with dye particles (Gashti et al., 2014; Radha et al., 2016).

Coconut is a dye yielding whose botanical name is Cocos nucifera. The coloring part of coconut coir is a tannin dye (Figure 1a) used to dye cotton, wool and silk (Adeel et al., 2020a; Teli and Pandit, 2017). The extract of coconut coir possesses antifungal and antimicrobial properties (Uy et al., 2019).
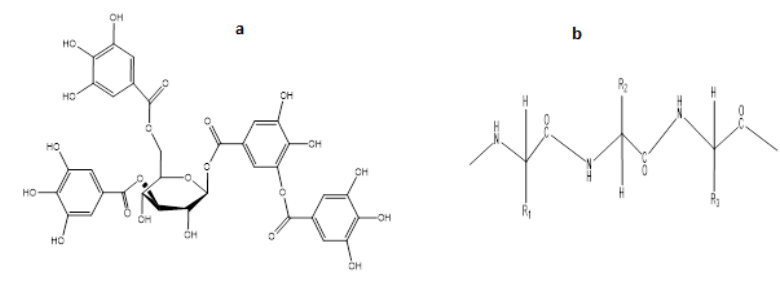

Figure 1. Tannin structure (a) Functional unit of wool fabric (b) The aims of current study were

(i) To isolate natural dye from coconut coir under microwave irradiation in different extraction media

(ii) Microwave-assisted dyeing of wool fabric following the optimization of different dyeing variables

(iii) The effect of different chemical mordants \& biomordants on the colour characteristics of fabric dyed with optimized coconut coir extract

\section{Materials and methods}

\subsection{Availability of material}

For the dyeing process, coconut coir has been purchased from the local market of Faisalabad, Pakistan. Wool fabric was purchased from the textile market in Faisalabad, Pakistan for dyeing. Chemical and herbal based biomordants were also purchased from the herbal market in Faisalabad, Pakistan. Coconut coir was washed with water to remove dust particles and after necessary processes, the uniform particle size powder was obtained and stored in jars for further isolation processes.

\subsection{Extraction of colorant}

For isolation of colorant from coconut coir, $4 \mathrm{~g}$ of coconut coir powder was dissolved in $100 \mathrm{~mL}$ of water and was heated for 45 minutes at boiling temperature keeping ( $M$ : $L$ of $1: 25)$. After heating, the mixture was filtered and filtrate was stored. Similarly, plant extracts were prepared in alkaline, methanol and acidic methanol. Each extract of coconut coir and pre-treated wool fabrics were exposed to microwave irradiation for $1,2,3,4,5$ and 6 minutes (Adeel et al., 2020b).

\subsection{Optimization of dyeing process under Microwave treatment}

Dyeing of pre-treated wool fabric was done using irradiated (RE) and non-irradiated extract (NRE) with irradiated wool (RW) and non-irradiated wool (NRW) for 45 min. at $85^{\circ} \mathrm{C}$ using $\mathrm{NaCl}$ as an electrolyte following the continuous stirring. After dyeing, the wool fabric was washed to remove the unfixed dye. Color strength values were evaluated using spectra flash SF600.

\subsubsection{Optimization of dyeing time}

The dyeing contact time is a crucial parameter as it defines the point where maximum absorption was attained. For it, non-irradiated wool fabric was dyed for a range of dyeing time periods (25-85 min.) using irradiated optimal extract. Dyed wool fabrics were rinsed with water to remove the free dye and other chemicals and $t$ color strength was measured.

\subsubsection{Dye bath $\mathrm{pH}$}

The optimal extract was used to dye the wool with liquor ratio $1: 25$ at different $\mathrm{pH}$ (1-9) values and temperature was $85^{\circ} \mathrm{C}$ at $45 \mathrm{~min}$. using the same methodology described earlier. After dyeing, the wool fabric was exposed to find $\mathrm{K} / \mathrm{S}$.

\subsubsection{Dye bath temperature}

The optimal extract was used to dye the wool with liquor ratio $1: 25$ at different temperatures $\left(25-85^{\circ} \mathrm{C}\right)$ at $45 \mathrm{~min}$. using the same methodology described earlier. After dyeing, the wool fabric was washed to remove the access dye and other chemicals, dried and were used to find the colour strength values.

\subsubsection{Effect of Salts on dyeing}

Salts act as exhausting agents in dyeing process. For current study, table $(\mathrm{NaCl})$ and Gluber $\left(\mathrm{Na}_{2} \mathrm{SO}_{4}\right)$ salts were added in different proportions $(1-11 \mathrm{~g} / 100 \mathrm{~mL})$ during dyeing at $80{ }^{\circ} \mathrm{C}$ for 45 minutes. After dyeing, the wool fabric was washed to remove the access dye and other 
chemicals, dried and were used to find the colour strength values (Kadam et al., 2019)

\subsection{Dyeing impact with mordants}

Shade quality and color ratings could be improved by using various mordants. Here ten mordants were employed. Mordants make firm shade, via metal dye complex formation. For pre-mordanting process, optimal wool fabrics were mordanted using solutions (1$11 \mathrm{~g} / 100 \mathrm{~mL}$ ) of salts of aluminium, cobalt, copper, iron, tin \& Tannic acid at $80{ }^{\circ} \mathrm{C}$ for $45 \mathrm{~min}$. For comparative study and make process more sustainable, four bio-mordants i.e. solutions $(1-11 \mathrm{~g} / 100 \mathrm{~mL})$ of Acacia, Henna, Pomegranate and Turmeric as bio-mordants were employed. The samples were subjected for $\mathrm{K} / \mathrm{S}$ (colour strength) as well as colour fastness properties. For postmordanting the fabrics were dyed at $80^{\circ} \mathrm{C}$ for 45 minutes and then subjected to mordanting using Alum, Cobalt, Copper, Iron, Tannic acid and Tin as chemical mordant, whereas Acacia, Henna, Pomegranate and Turmeric as bio-mordants (Adeel et al., 2019; Shahid et al., 2017).

Table 1a. Fastness grading of wool fabric dyed with coconut coir extract before chemical mordanting

\begin{tabular}{|c|c|c|c|c|c|c|c|c|c|}
\hline \multirow{2}{*}{\multicolumn{3}{|c|}{ Pre-chemical Mordant }} & \multicolumn{7}{|c|}{ Concentration of mordants (\%) } \\
\hline & & & Control & 1 & 3 & 5 & 7 & 9 & 11 \\
\hline \multirow{7}{*}{ Al } & \multicolumn{2}{|c|}{ LF } & $3-4$ & $4-5$ & $4-5$ & $4-5$ & $4-5$ & $4-5$ & $4-5$ \\
\hline & \multicolumn{2}{|c|}{ WF } & 3 & $4-5$ & $4-5$ & $4-5$ & $4-5$ & $4-5$ & $4-5$ \\
\hline & \multicolumn{2}{|c|}{ DRF } & $3-4$ & $4-5$ & $4-5$ & $4-5$ & $4-5$ & $4-5$ & $4-5$ \\
\hline & \multicolumn{2}{|c|}{ WRF } & 3 & $4-5$ & $4-5$ & $4-5$ & $4-5$ & $4-5$ & $4-5$ \\
\hline & \multicolumn{2}{|c|}{ Dry Cleaning } & $3-4$ & 4 & 4 & 4 & 4 & $4-5$ & 4 \\
\hline & \multirow{2}{*}{ Perspiration } & Acidic & $3-4$ & 4 & 4 & 4 & $4-5$ & 5 & 4 \\
\hline & & Basic & $3-4$ & 4 & 4 & 4 & $4-5$ & 5 & 4 \\
\hline \multirow{7}{*}{$\mathrm{Fe}$} & \multicolumn{2}{|c|}{ LF } & $3-4$ & $4-5$ & $4-5$ & $4-5$ & $4-5$ & 5 & $4-5$ \\
\hline & \multicolumn{2}{|c|}{ WF } & $3-4$ & $4-5$ & $4-5$ & $4-5$ & $4-5$ & 5 & $4-5$ \\
\hline & \multicolumn{2}{|c|}{ DRF } & 3 & 5 & 5 & 5 & 5 & 5 & 5 \\
\hline & \multicolumn{2}{|c|}{ WRF } & $3-4$ & $4-5$ & $4-5$ & $4-5$ & $4-5$ & 5 & $4-5$ \\
\hline & \multicolumn{2}{|c|}{ Dry Cleaning } & 3 & $4-5$ & $4-5$ & $4-5$ & $4-5$ & 5 & $4-5$ \\
\hline & \multirow{2}{*}{ Perspiration } & Acidic & $3-4$ & $4-5$ & $4-5$ & $4-5$ & $4-5$ & 5 & $4-5$ \\
\hline & & Basic & $3-4$ & $4-5$ & $4-5$ & $4-5$ & $4-5$ & 5 & $4-5$ \\
\hline \multirow{7}{*}{ Tannic Acid } & \multicolumn{2}{|c|}{$\mathrm{LF}$} & $3-4$ & $4-5$ & $4-5$ & $4-5$ & 5 & $4-5$ & $4-5$ \\
\hline & \multicolumn{2}{|c|}{ WF } & $3-4$ & 4 & 4 & 4 & 5 & 4 & 4 \\
\hline & \multicolumn{2}{|c|}{ DRF } & 3 & 5 & 5 & 5 & 5 & 5 & 5 \\
\hline & \multicolumn{2}{|c|}{ WRF } & $3-4$ & $4-5$ & $4-5$ & $4-5$ & 5 & $4-5$ & $4-5$ \\
\hline & \multicolumn{2}{|c|}{ Dry Cleaning } & 3 & $4-5$ & $4-5$ & $4-5$ & 5 & $4-5$ & $4-5$ \\
\hline & \multirow{2}{*}{ Perspiration } & Acidic & $3-4$ & $4-5$ & $4-5$ & $4-5$ & 5 & $4-5$ & $4-5$ \\
\hline & & Basic & $3-4$ & $4-5$ & $4-5$ & $4-5$ & 5 & $4-5$ & $4-5$ \\
\hline
\end{tabular}

Table 1b. Fastness grading of wool fabric dyed with coconut coir extract after chemical mordanting

\begin{tabular}{|c|c|c|c|c|c|c|c|c|c|}
\hline \multirow{2}{*}{\multicolumn{3}{|c|}{ Post-chemical Mordant }} & \multicolumn{7}{|c|}{ Percentage of mordants (\%) } \\
\hline & & & Control & 1 & 3 & 5 & 7 & 9 & 11 \\
\hline \multirow{7}{*}{ Al } & \multicolumn{2}{|c|}{$\mathrm{LF}$} & $3-4$ & $4-5$ & $4-5$ & 5 & $4-5$ & $4-5$ & $4-5$ \\
\hline & \multicolumn{2}{|c|}{ WF } & 3 & $4-5$ & $4-5$ & 5 & $4-5$ & $4-5$ & $4-5$ \\
\hline & \multicolumn{2}{|c|}{ DRF } & $3-4$ & $4-5$ & $4-5$ & 5 & $4-5$ & $4-5$ & $4-5$ \\
\hline & \multicolumn{2}{|c|}{ WRF } & 3 & $4-5$ & $4-5$ & 5 & $4-5$ & $4-5$ & $4-5$ \\
\hline & \multicolumn{2}{|c|}{ Dry Cleaning } & $3-4$ & 4 & 4 & 5 & 4 & 4 & 4 \\
\hline & \multirow{2}{*}{ Perspiration } & Acidic & $3-4$ & 4 & 4 & 5 & 4 & 4 & 4 \\
\hline & & Basic & $3-4$ & 4 & 4 & 5 & 4 & 4 & 4 \\
\hline \multirow{7}{*}{$\mathrm{Fe}$} & \multicolumn{2}{|c|}{ LF } & $3-4$ & $4-5$ & $4-5$ & $4-5$ & $4-5$ & $4-5$ & 5 \\
\hline & \multicolumn{2}{|c|}{ WF } & $3-4$ & $4-5$ & $4-5$ & $4-5$ & $4-5$ & $4-5$ & 5 \\
\hline & \multicolumn{2}{|c|}{ DRF } & 3 & 5 & 5 & 5 & 5 & 5 & 5 \\
\hline & \multicolumn{2}{|c|}{ WRF } & $3-4$ & $4-5$ & $4-5$ & $4-5$ & $4-5$ & $4-5$ & 5 \\
\hline & \multicolumn{2}{|c|}{ Dry Cleaning } & 3 & $4-5$ & $4-5$ & $4-5$ & $4-5$ & $4-5$ & 5 \\
\hline & \multirow{2}{*}{ Perspiration } & Acidic & $3-4$ & $4-5$ & $4-5$ & $4-5$ & $4-5$ & $4-5$ & 5 \\
\hline & & Basic & $3-4$ & $4-5$ & $4-5$ & $4-5$ & $4-5$ & $4-5$ & 5 \\
\hline \multirow{7}{*}{ Tannic Acid } & \multicolumn{2}{|c|}{$\mathrm{LF}$} & $3-4$ & $4-5$ & $4-5$ & $4-5$ & 5 & $4-5$ & $4-5$ \\
\hline & \multicolumn{2}{|c|}{ WF } & $3-4$ & 4 & 4 & 4 & 5 & 4 & 4 \\
\hline & \multicolumn{2}{|c|}{ DRF } & 3 & 5 & 5 & 5 & 5 & 5 & 5 \\
\hline & \multicolumn{2}{|c|}{ WRF } & $3-4$ & $4-5$ & $4-5$ & $4-5$ & 5 & $4-5$ & $4-5$ \\
\hline & \multicolumn{2}{|c|}{ Dry Cleaning } & 3 & $4-5$ & $4-5$ & $4-5$ & 5 & $4-5$ & $4-5$ \\
\hline & \multirow{2}{*}{ Perspiration } & Acidic & $3-4$ & $4-5$ & $4-5$ & $4-5$ & 5 & $4-5$ & $4-5$ \\
\hline & & Basic & $3-4$ & $4-5$ & $4-5$ & $4-5$ & 5 & $4-5$ & $4-5$ \\
\hline
\end{tabular}


Table 2a. Fastness grading of wool fabric dyed with coconut coir extract before biomordanting

\begin{tabular}{|c|c|c|c|c|c|c|c|c|c|}
\hline \multirow{2}{*}{\multicolumn{3}{|c|}{ Pre-bio Mordant }} & \multicolumn{7}{|c|}{ Percentage of mordants (\%) } \\
\hline & & & Control & 1 & 3 & 5 & 7 & 9 & 11 \\
\hline \multirow{7}{*}{ Acacia bark } & \multicolumn{2}{|c|}{ LF } & $3-4$ & $4-5$ & $4-5$ & 5 & $4-5$ & $4-5$ & $4-5$ \\
\hline & \multicolumn{2}{|c|}{ WF } & 3 & $4-5$ & $4-5$ & 5 & $4-5$ & $4-5$ & $4-5$ \\
\hline & \multicolumn{2}{|c|}{ DRF } & $3-4$ & $4-5$ & $4-5$ & 5 & $4-5$ & $4-5$ & $4-5$ \\
\hline & \multicolumn{2}{|c|}{ WRF } & 3 & $4-5$ & $4-5$ & 5 & $4-5$ & $4-5$ & $4-5$ \\
\hline & \multicolumn{2}{|c|}{ Dry Cleaning } & $3-4$ & $4-5$ & $4-5$ & 5 & $4-5$ & $4-5$ & $4-5$ \\
\hline & \multirow{2}{*}{ Perspiration } & Acidic & $3-4$ & $4-5$ & $4-5$ & 5 & $4-5$ & $4-5$ & $4-5$ \\
\hline & & Basic & $3-4$ & $4-5$ & $4-5$ & 5 & $4-5$ & $4-5$ & $4-5$ \\
\hline \multirow{7}{*}{ Henna } & \multicolumn{2}{|c|}{ LF } & $3-4$ & $4-5$ & $4-5$ & $4-5$ & 5 & $4-5$ & $4-5$ \\
\hline & \multicolumn{2}{|c|}{ WF } & $3-4$ & $4-5$ & $4-5$ & $4-5$ & 5 & $4-5$ & $4-5$ \\
\hline & \multicolumn{2}{|c|}{ DRF } & 3 & $4-5$ & $4-5$ & $4-5$ & 5 & $4-5$ & $4-5$ \\
\hline & \multicolumn{2}{|c|}{ WRF } & $3-4$ & $4-5$ & $4-5$ & $4-5$ & 5 & $4-5$ & $4-5$ \\
\hline & \multicolumn{2}{|c|}{ Dry Cleaning } & 3 & $4-5$ & $4-5$ & $4-5$ & 5 & $4-5$ & $4-5$ \\
\hline & \multirow{2}{*}{ Perspiration } & Acidic & $3-4$ & $4-5$ & $4-5$ & $4-5$ & 5 & $4-5$ & $4-5$ \\
\hline & & Basic & $3-4$ & $4-5$ & $4-5$ & $4-5$ & 5 & $4-5$ & $4-5$ \\
\hline \multirow{7}{*}{ Turmeric } & \multicolumn{2}{|c|}{ LF } & $3-4$ & 4 & 4 & $4-5$ & 4 & 4 & 4 \\
\hline & \multicolumn{2}{|c|}{ WF } & $3-4$ & 4 & 4 & $4-5$ & 4 & 4 & 4 \\
\hline & \multicolumn{2}{|c|}{ DRF } & 3 & 4 & 4 & $4-5$ & 4 & 4 & 4 \\
\hline & \multicolumn{2}{|c|}{ WRF } & $3-4$ & 4 & 4 & $4-5$ & 4 & 4 & 4 \\
\hline & \multicolumn{2}{|c|}{ Dry Cleaning } & 3 & 4 & 4 & $4-5$ & 4 & 4 & 4 \\
\hline & \multirow{2}{*}{ Perspiration } & Acidic & $3-4$ & 4 & 4 & $4-5$ & 4 & 4 & 4 \\
\hline & & Basic & $3-4$ & 4 & 4 & $4-5$ & 4 & 4 & 4 \\
\hline \multirow{7}{*}{ Pomegranate } & \multicolumn{2}{|c|}{$\mathrm{LF}$} & $3-4$ & $4-5$ & $4-5$ & $4-5$ & $4-5$ & $4-5$ & 5 \\
\hline & \multicolumn{2}{|c|}{ WF } & $3-4$ & $4-5$ & $4-5$ & $4-5$ & $4-5$ & $4-5$ & 5 \\
\hline & \multicolumn{2}{|c|}{ DRF } & 3 & $4-5$ & $4-5$ & $4-5$ & $4-5$ & $4-5$ & 5 \\
\hline & \multicolumn{2}{|c|}{ WRF } & $3-4$ & $4-5$ & $4-5$ & $4-5$ & $4-5$ & $4-5$ & 5 \\
\hline & \multicolumn{2}{|c|}{ Dry Cleaning } & 3 & $4-5$ & $4-5$ & $4-5$ & $4-5$ & $4-5$ & 5 \\
\hline & Percniration & Acidic & $3-4$ & $4-5$ & $4-5$ & $4-5$ & $4-5$ & $4-5$ & 5 \\
\hline & Perspiration & Basic & $3-4$ & $4-5$ & $4-5$ & $4-5$ & $4-5$ & $4-5$ & 5 \\
\hline
\end{tabular}

Table $\mathbf{2 b}$ b. Fastness grading of wool fabric dyed with coconut coir extract after biomordanting

\begin{tabular}{|c|c|c|c|c|c|c|c|c|c|}
\hline \multirow{2}{*}{\multicolumn{3}{|c|}{ Post-bio Mordant }} & \multicolumn{7}{|c|}{ Percentage of mordants (\%) } \\
\hline & & & Control & 1 & 3 & 5 & 7 & 9 & 11 \\
\hline \multirow{7}{*}{ Acacia bark } & \multicolumn{2}{|c|}{$\mathrm{LF}$} & $3-4$ & $4-5$ & $4-5$ & 5 & $4-5$ & $4-5$ & $4-5$ \\
\hline & \multicolumn{2}{|c|}{ WF } & 3 & $4-5$ & $4-5$ & 5 & $4-5$ & $4-5$ & $4-5$ \\
\hline & \multicolumn{2}{|c|}{ DRF } & $3-4$ & $4-5$ & $4-5$ & 5 & $4-5$ & $4-5$ & $4-5$ \\
\hline & \multicolumn{2}{|c|}{ WRF } & 3 & $4-5$ & $4-5$ & 5 & $4-5$ & $4-5$ & $4-5$ \\
\hline & \multicolumn{2}{|c|}{ Dry Cleaning } & $3-4$ & $4-5$ & $4-5$ & 5 & $4-5$ & $4-5$ & $4-5$ \\
\hline & \multirow{2}{*}{ Perspiration } & Acidic & $3-4$ & $4-5$ & $4-5$ & 5 & $4-5$ & $4-5$ & $4-5$ \\
\hline & & Basic & $3-4$ & $4-5$ & $4-5$ & 5 & $4-5$ & $4-5$ & $4-5$ \\
\hline \multirow{7}{*}{ Henna } & \multicolumn{2}{|c|}{ LF } & $3-4$ & $4-5$ & $4-5$ & $4-5$ & $4-5$ & $4-5$ & 5 \\
\hline & \multicolumn{2}{|c|}{ WF } & $3-4$ & $4-5$ & $4-5$ & $4-5$ & $4-5$ & $4-5$ & 5 \\
\hline & \multicolumn{2}{|c|}{ DRF } & 3 & $4-5$ & $4-5$ & $4-5$ & $4-5$ & $4-5$ & 5 \\
\hline & \multicolumn{2}{|c|}{ WRF } & $3-4$ & $4-5$ & $4-5$ & $4-5$ & $4-5$ & $4-5$ & 5 \\
\hline & \multicolumn{2}{|c|}{ Dry Cleaning } & 3 & $4-5$ & $4-5$ & $4-5$ & $4-5$ & $4-5$ & 5 \\
\hline & \multirow{2}{*}{ Perspiration } & Acidic & $3-4$ & $4-5$ & $4-5$ & $4-5$ & $4-5$ & $4-5$ & 5 \\
\hline & & Basic & $3-4$ & $4-5$ & $4-5$ & $4-5$ & $4-5$ & $4-5$ & 5 \\
\hline \multirow{7}{*}{ Turmeric } & \multicolumn{2}{|c|}{ LF } & $3-4$ & 4 & 4 & 4 & 4 & 4 & $4-5$ \\
\hline & \multicolumn{2}{|c|}{ WF } & $3-4$ & 4 & 4 & 4 & 4 & 4 & $4-5$ \\
\hline & \multicolumn{2}{|c|}{ DRF } & 3 & 4 & 4 & 4 & 4 & 4 & $4-5$ \\
\hline & \multicolumn{2}{|c|}{ WRF } & $3-4$ & 4 & 4 & 4 & 4 & 4 & $4-5$ \\
\hline & \multicolumn{2}{|c|}{ Dry Cleaning } & 3 & 4 & 4 & 4 & 4 & 4 & $4-5$ \\
\hline & \multirow{2}{*}{ Perspiration } & Acidic & $3-4$ & 4 & 4 & 4 & 4 & 4 & $4-5$ \\
\hline & & Basic & $3-4$ & 4 & 4 & 4 & 4 & 4 & $4-5$ \\
\hline \multirow{7}{*}{ Pomegranate } & \multicolumn{2}{|c|}{$\mathrm{LF}$} & $3-4$ & $4-5$ & $4-5$ & 5 & $4-5$ & $4-5$ & $4-5$ \\
\hline & \multicolumn{2}{|c|}{ WF } & $3-4$ & $4-5$ & $4-5$ & 5 & $4-5$ & $4-5$ & $4-5$ \\
\hline & \multicolumn{2}{|c|}{ DRF } & 3 & $4-5$ & $4-5$ & 5 & $4-5$ & $4-5$ & $4-5$ \\
\hline & \multicolumn{2}{|c|}{ WRF } & $3-4$ & $4-5$ & $4-5$ & 5 & $4-5$ & $4-5$ & $4-5$ \\
\hline & \multicolumn{2}{|c|}{ Dry Cleaning } & 3 & $4-5$ & $4-5$ & 5 & $4-5$ & $4-5$ & $4-5$ \\
\hline & Persnir & Acidic & $3-4$ & $4-5$ & $4-5$ & 5 & $4-5$ & $4-5$ & $4-5$ \\
\hline & Perspiration & Basic & $3-4$ & $4-5$ & $4-5$ & 5 & $4-5$ & $4-5$ & $4-5$ \\
\hline
\end{tabular}




\subsection{Analysis of dyed and undyed fabrics}

All the fabrics dyed after extraction of colorant, during dyeing conditions as well as shade developments processes were assessed for colour strength $(\mathrm{K} / \mathrm{S})$ values through the standard equation called Kubelka-Munk equation integrated in software by data color (Spectraflash spectrophotometer SF600, USA). Colorfastness ratings of dyed chemical and bio-mordanted wool fabric at optimum conditions were achieved at grey scale after their exposure under ISO Standards methods of light, washing and rubbing (Adeel et al., 2019). FTIR analysis was done to investigate the influence of microwave treatment on the particular peak of functional unit of wool fibers. This analysis uses infrared light to scan and observe the chemical properties of samples. Samples were prepared using optimum conditions under irradiated microwave treatment. Control sample was also analyzed by FTIR for comparison purpose.

\section{Results and discussion}

\subsection{Dye extraction process}

The results shown in Figure 2(a-d) reveal that along with solvent, irradiation has also played a unique role in isolation of colorant. During isolation two factors have worked simultaneously i.e. rupture of cell wall to make good interaction of biological potent molecule (tannin) with solvent $\&$ the tunning of fabric functional site (amido linkage), which has added value in coloration to give desired results. The results also show that methanolic medium due to its excellent polarizability and interaction power has interacted well with tannin as compared to other solvents used (Deng et al., 2019).

The isolation of colorant (RE) using microwave (MW) treatment for $2 \mathrm{~min}$. has given high color strength onto irradiated wool (RW). Higher irradiation level can cause possibility of other functional molecules to interact with solvent thereby dominating over the actual colorant to give lower results, whereas due to less tuned surface the unirradiated fabric (NRW) also may sorb less colorant to give lower results (Jabeen et al., 2019). Hence overall, for getting effective color yield, irradiated extract (RE, 2 min) should be used for dyeing of irradiated fabric (RW, $2 \mathrm{~min}$ ) to get excellent results.

\subsection{Optimization of dyeing parameters}

\subsection{1. $\mathrm{pH}$ of dye bath}

Extract $\mathrm{pH}$ is of great importance because at particular level, excellent results are obtained. The nature of fabric, its functional site as well as colorant nature \& stability also play their role (Safapour et al., 2018). It can be seen from Figure 3(a) that dye bath of $\mathrm{pH} 1$ has given excellent results whereas moving towards higher $\mathrm{pH}(\mathrm{pH}>1)$, the color strength was decreased. This is because wool fabric containing amido linkage force protontion under acidic conditions $(\mathrm{pH}<3)$, due to which active sites are available for bonding (Jiang et al., 2019). Whereas due to higher $\mathrm{pH}$ $(\mathrm{pH}>3)$, the certain sites are not available to give good color depth (Rani et al., 2020). Hence overall, irradiated coconut coir extract of $\mathrm{pH} 1$ should be used to color irradiated wool (RW) for obtaining desired results.

\subsubsection{Dye bath temperature}

The Figure $3(\mathrm{~b})$ shows that MW treated wool fabric (RW) dyed at $65^{\circ} \mathrm{C}$ has given excellent results when dye bath of $1 \mathrm{pH}$ was employed. At low temperatures, the dye molecules do not move towards the fabric surface whereas high heating may have caused degradation of dye molecules which were not sorbed on the fabric properly thereby resulting into low color strength (Samanta, 2020). Hence, it can be seen that irradiation treatment has leveled heating point $\left(65^{\circ} \mathrm{C}\right)$ of the dyeing of woolen fabric using methanolic coconut coir extract which show it is an energy effective tool (Alebeid et al., 2020).

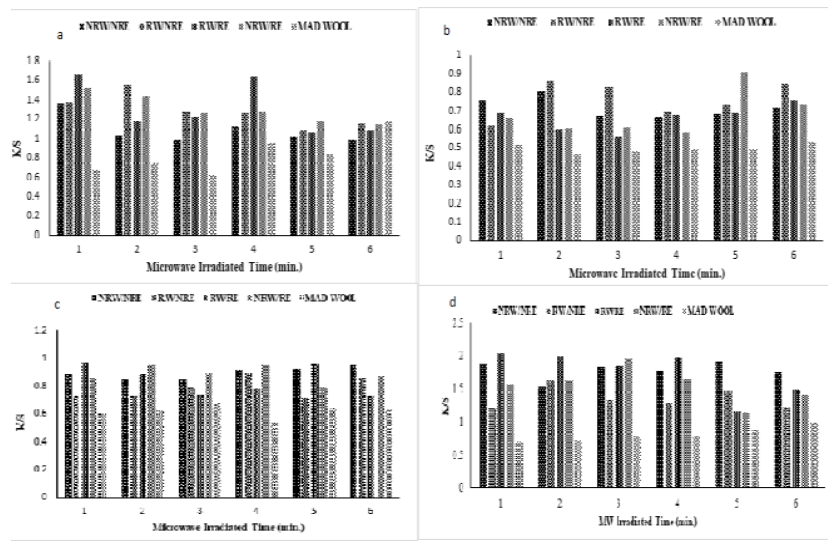

Figure 2. Microwave treatment of pre-treated silk dyeing by aqueous extract (a) acidic extract (b) Alkaline extract (c) methanolic extract (d) of coconut coir NRS: Non-irradiated silk NRE: Non-irradiated extract RS: Irradiated silk RE: Irradiated extract

\subsubsection{Effect of dyeing time on wool dyeing}

In dyeing process, time parameter plays a significant role on the fabric surface. Wool has a $-\mathrm{COOH}$ group which takes suitable time for firm bonding with colorant to give good color strength (Samanta, 2020). Furthermore, dye molecules in clusters also take time to break into small size for a better sorption process. Figure 3(c) shows that the optimal time for wool dyeing is $65 \mathrm{~min}$. Less contact time may not contact cause effective acceleration of molecules, whereas too much heating contact (85 min) with dye bath may cause hydrolytic degradation thereby causing poor fixation (Nonso et al., 2019). Overall, dyeing of irradiated fabric for $65 \mathrm{~min}$. should be done using coconut coir extract. Again the shortening of dye time reveals that irradiation tool is time effective tool in nature.

\subsubsection{Effect of salt concentration}

Direct dyeing of wool fabric without salt addition decreases the color strength due to poor exhaustion. Salt enhances the color properties of the wool fabric during dyeing due to creation of ionic atmosphere around fabric, dye \& water molecules (Nonso et al., 2019). Figure 3(d) shows that the concentration of $5 \mathrm{~g} / 100 \mathrm{~mL}$ of Glauber salt 
\& Table salt enhances the color strength of the wool dyed with coconut coir extract. Overall, Glauber salt has given excellent exhaustion whereas too smaller salt may cause over exhaustion which leads to poor color fixation either by resulting into low color strength (K/S) (Jabar et al., 2020). Hence, again it can be seen that irradiation of fabric \& extract has revealed the utilization of salt which reveals that irradiation tool is cost effective in nature.

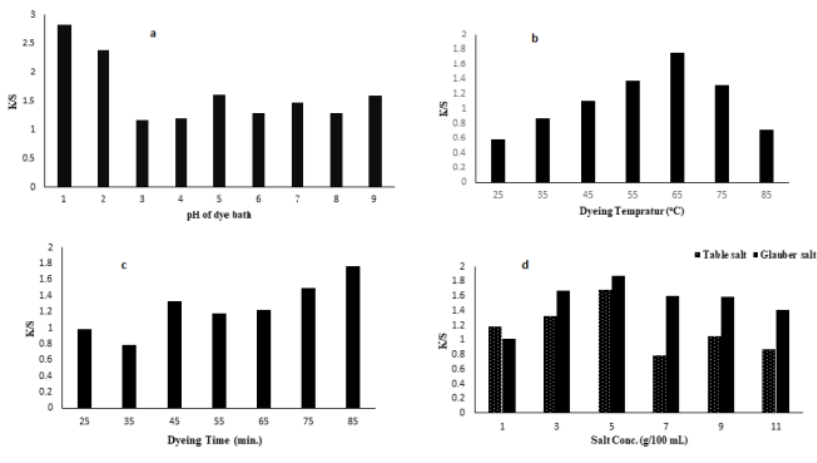

Figure 3. Effect of dyeing pH (a) Dyeing temperature (b) Dyeing time (c) \& Salt concentrations (d) on dyeing of wool with coconut coir extract

\subsection{The behavior of different mordants on optimal dyed wool}

Mordanting is an important tool for improvement in color fastness properties as well as introducing new shades. Biomordants are newly introduced anchors for this place in place of some chemical mordants like salts of $\mathrm{Cr}, \mathrm{Cd}, \mathrm{Ni}$, $\mathrm{Cu}$ etc. In this study, some of biomordants have been introduced along with sustainable chemical anchors such as salts of $\mathrm{Al} \& \mathrm{Fe}$, and tannic acid (TA). The results given in Figure 4(a) for pre-chemical \& pre-biomordants reveal that $\mathrm{Fe}$ as chemical mordants has given better results (Nonso et al., 2019) whereas pomegranate having tannin has given excellent results. Similarly Figure 4(b) for chemical mordanting show that Fe as post chemical \& for biomordanting show that turmeric containing curcumin as post biomordant has given desired results. This is because Al, Fe \& tannic acid (TA) form metal dye complex which form stable shades with good color strength (Rani et al., 2020). All biomordants have $-\mathrm{OH}$ group which via making extra $\mathrm{H}$-bonding with colorant \& fabric given excellent results (Alebeid et al., 2020, 2020c). But overall biomordants have shown excellent potential to make process green and sustainable $\&$ viable in natural dyeing process.
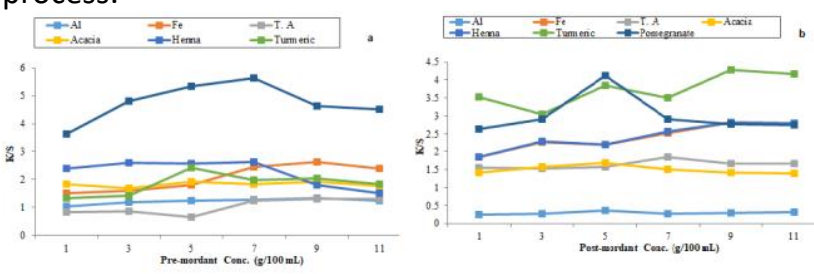

Figure 4. Effect of Pre-chemical and bio-mordants (a) \& Postchemical and bio-mordants (b) Post-mordant on dyeing of wool with coconut extract

\subsection{Color rating values}

The fastness properties are important characteristics of any dyed fabric particularly in natural dyeing process. For the present $\mathrm{MW}$ rays as an isolation tool, biomordants as shade development with excellent fastness properties have been employed. The results show that biomordants have enhanced the rating from good to excellent (Table $2 a$ $\&$ b), whereas in comparison metals used as chemical mordants have also given good ratings (Table $1 \mathrm{a} \& \mathrm{~b}$ ). This good rating is due to formation of extra $\mathrm{H}$-bonding, conjugation system of biomordants \& colourant as well as mode of attachment (Khan et al., 2016; Adeel et al., 2019). Similarly using metal electrolytes, the firm fixation of metal dye complex onto fabric, reduction of metal as well as mode of attachment has played their role. Hence, overall sustainable shade development tool i.e. biomordanting has given desired ratings whereas addition of $\mathrm{MW}$ rays has valorized natural dyeing of wool with coconut coir based tannin natural dye (Adeel et al., 2020c; Islam, 2017).

\subsection{FTIR analysis}

FTIR analysis of untreated silk fabric and microwave treated wool fabric FTIR dyed with natural tannin dye extracted from Coconut coir are given in Figure 5. As can be seen from Figure 5, the FTIR curve of the microwavetreated wool was changed compared to the untreated wool. According to FTIR spectra, the bonds between C-C, $\mathrm{C}-\mathrm{N}$, and $\mathrm{N}-\mathrm{H}$ stress were shown ranged from $1630.08 \mathrm{~cm}^{-}$ $1,1512.71 \mathrm{~cm}^{-1}, 1227.10 \mathrm{~cm}^{-1}$ depicting that $\mathrm{MW}$ radiation has a significant effect on the chemical structure of wool fibers.

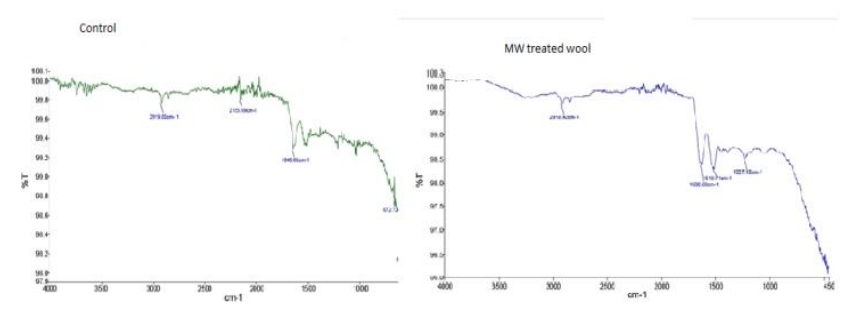

Figure 5. FTIR of untreated wool and microwave treated wool

\section{Conclusions}

It has been found that microwave treatment during extraction of natural dye from coconut coir and dyeing of wool has the potential to dye wool fabric being ecofriendly, green, less cost and less time effective process. The research concluded that the extract of natural dye (tannin base) from coconut coir by mcrowave treatment with the dyeing of chemical and biomordanting of wool fabric can be utilized as reddishbrown color as compare to synthetic dyes which more hazards for the environment and not eco-friendly. Methanolic extract of coconut coir at $\mathrm{pH} 1$ for $1 \mathrm{~min}$. Microwave treatment using $5 \mathrm{~g} / 100 \mathrm{~mL} \mathrm{Na}_{2} \mathrm{SO}_{4}$ gives good color strength and darker shade by dyeing unirradiated wool fabric for $65 \mathrm{~min}$. at $65^{\circ} \mathrm{C}$. Chemical mordant (Al, Fe and Tannic acid) and bio-mordant such as Henna, Pomegranate, Acacia and Turmeric also applied to improve the color strength and make strong bonding with wool fabric but bio mordants like Pomegranate (6\%) in 
pre-mordanting and Turmeric (6\%) in post-mordanting gave the excellent color and have given high $\mathrm{K} / \mathrm{S}$ values. Furthermore, Microwave treatment has also the tendency to improve the color strength on wool using coconut coir extract as a natural dye under optimum conditions. More that, using bio-mordants is not hazard for heath, ecofriendly, green, eco-label and cheaper for the dyeing process.

\section{Declaration of conflicting interests}

All the authors of this paper declare no conflict of interest.

\section{Funding}

The current study has been funded by UNESCO via PhosAgro/UNESCO/IUPAC/GCUF Project No. 128, which was carried out in Department of Applied Chemistry, Government College University, Faisalabad, Pakistan.

\section{References}

Abrar S., Naqvi K.R., Javed S., Kiran S., Qaiser I., Gulzar T. and Abbas Q. (2020), Investigation of the Physiochemical Properties of Beauty Soaps Available in Pakistan, Tenside Surfactants Detergents, 57(2), 162-167.

Adeel S., Kiran S., Ahmad T., Habib N., Tariq K. and Hussaan M. (2020a), Bio-Mordants in Conjunction With Sustainable Radiation Tools for Modification of Dyeing of Natural Fibers, Frontiers of Textile Materials, 355-367.

Adeel S., Kiran S., Habib N., Hassan A., Kamal S., Qayyum M.A. and Tariq K. (2020b), Sustainable ultrasonic dyeing of wool using coconut coir extract, Textile Research Journal, 90(7-8), 744-756.

Adeel S., Rehman F.U., Hameed A., Habib N., Kiran S., Zia K.M. and Zuber M. (2020c), Sustainable extraction and dyeing of microwave-treated silk fabric using arjun bark colorant, Journal of Natural Fibers, 17(5), 745-758.

Adeel S., Rehman F.U., Iqbal M.U., Habib N., Kiran S., Zuber M., Zia K.M. and Hameed A. (2019), Ultrasonic assisted sustainable dyeing of mordanted silk fabric using arjun (Terminalia arjuna) bark extracts, Environmental Progress \& Sustainable Energy, 38(s1), S331-S339.

Alebeid O.K., Pei L., Zhou W. and Wang J. (2020), Sustainable wool fibers dyeing using henna extract in non-aqueous medium, Environmental Chemistry Letters, 18(2), 489-494.

Ali K.M., Islam S.U. and Mohammad F. (2016), Extraction of natural dye from walnut bark and its dyeing properties on wool yarn, Journal of Natural Fibers, 13(4), 458-469.

Baaka N., Mahfoudhi A., Haddar W., Mhenni M.F. and Mighri Z. (2016), Green dyeing process of modified cotton fibres using natural dyes extracted from Tamarix aphylla (L.) Karst. leaves, Natural Product Research, 31(1), 22-31.

Deng B., Liu Z. and Zou Z. (2019), Optimization of microwaveassisted extraction saponins from Sapindus mukorossi Pericarps and an evaluation of their inhibitory activity on Xanthine Oxidase, Journal of Chemistry, 2019, 1-11.

Ebrahimi I. and Gashti P.M. (2015), Extraction of juglone from Pterocarya fraxinifolia leaves for dyeing, anti-fungal finishing, and solar UV protection of wool, Coloration Technology, 131(6), 451-457.

Ebrahimi I. and Gashti P.M. (2016), Extraction of polyphenolic dyes from henna, pomegranate rind, and Pterocarya fraxinifolia for nylon 6 dyeing, Coloration Technology, 132(2), 162-176.

Gashti P.M., Katozian B., Shaver M. and Kiumarsi A. (2014), Clay nanoadsorbent as an environmentally friendly substitute for mordants in the natural dyeing of carpet piles, Coloration Technology, 130(1), 54-61.

Haddar W., Ben T.M., Meksi N. and Guesmi A. (2018), Application of anthocyanins as natural dye extracted from Brassica oleracea L. var. capitata f. rubra: dyeing studies of wool and silk fibres, Natural Product Research, 32(2), 141148.

Islam S.U. (2017), Plant-based Natural Products: Derivatives and Applications, John Wiley \& Sons.

Jabar J.M., Ogunmokun A.I. and Taleat T.A.A. (2020), Color and fastness properties of mordanted Bridelia ferruginea $B$ dyed cellulosic fabric, Fashion and Textiles, 7(1), 1-13.

Jabeen S., Ali S., Nadeem M., Arif K., Qureshi N., Shar G.A. and Siddiqua U.H. (2019), Statistical Modeling for the Extraction of Dye from Natural Source and Industrial Applications, Polish Journal of Environmental Studies, 28(4), 2145-2150.

Jiang H., Hu X., Meena B.I., Khan A., Hussain M.T., Yao J. and Wang J. (2019), Extraction of natural dyes from the stem of Caulis spatholobi and their application on wool, Textile Research Journal, 89(23-24), 5209-5217.

Kadam S., Sharma A., Islam S.U., Bramhecha I. and Sheikh J. (2019), Utilization of rice straw as a source of biomolecules for sustainable multifunctional finishing vis a vis dyeing of wool, Journal of Natural Fibers, 1-11.

Karadag R., Buyukakinci B.Y. and Torgan E. (2020), Extraction and Natural Cotton Dyeing of Valonia Oak and Anatolian Buckthorn by Microwave Irradiation, Journal of Natural Fibers, 1-14.

Kashyap R., Sharma N. and Sharma L. (2016), Dyeing of Cotton with Natural Dye Extract from Coconut Husk, International Journal of Science Technology \& Engineering, 3(4), 92-95.

Kiumarsi A., Gashti P.M., Salehi P. and Dayeni M. (2017), Extraction of dyes from Delphinium zalil flowers and dyeing silk yarns, The Journal of the Textile Institute, 108(1), 66-70.

Mansour R., Mighri Z. and Mhenni F. (2016), Exploring the potential uses of Vitis vinifera $L$. leaves as raw material for textile dyeing without metal mordants, Fibers \& Polymers, 17, 1621-26.

Nonso O.S., Genevieve O., Chinedu O., Onyinyechi N. and Kenneth O.C. (2019), Effect of Temperature and Mordant on the Dyeing of Cotton Using Sodium Hydroxide Extract of Whitfieldia lateritia Dye, International Journal of Innovative Research in Science, Engineering and Technology, 8(6), 73017308.

Safapour S., Sadeghi-Kiakhani M. and Eshaghloo-Galugahi S. (2018), Extraction, Dyeing, and antibacterial properties of crataegus elbursensis fruit natural dye on wool yarn, Fibers and Polymers, 19(7), 1428-1434.

Samanta P. (2020), A Review on Application of Natural Dyes on Textile Fabrics and Its Revival Strategy, In Chemistry and Technology of Natural and Synthetic Dyes and Pigments, IntechOpen.

Shabbir M., Islam S.U., Bukhari M.N., Rather L.J., Khan M.A. and Mohammad F. (2017), Application of Terminalia chebula natural dye on wool fiber-evaluation of color and fastness properties, Textiles and Clothing Sustainability, 2(1), 1-9.Teli 
M.D. and Pandit P. (2017), Novel method of ecofriendly single bath dyeing and functional finishing of wool protein with coconut shell extract biomolecules, ACS Sustainable Chemistry \& Engineering, 5(9), 8323-8333.

Unal F., Avinc O., Yavas A., Eren H.A. and Eren S. (2020), Contribution of UV Technology to Sustainable Textile Production and Design, Sustainability in the Textile and Apparel Industries, 163-187.

Uy I.A., Dapar M.L.G., Aranas A.T., Mindo R.A.R., Manting M.M.E., Torres M.A.J. and Demayo C.G. (2019), Qualitative assessment of the antimicrobial, antioxidant, phytochemical properties of the ethanolic extracts of the roots of Cocos nucifera L., Pharmacophore, 10, 63-75.

Yusuf M., Islam S.U., Khan M.A. and Mohammad F. (2016), Investigations of the colourimetric and fastness properties of wool dyed with colorants extracted from Indian madder using reflectance spectroscopy, Optik-International Journal for Light and Electron Optics, 127, 6087-93.

Yusuf M., Shabbir M. and Mohammad F. (2017), Natural colorants: Historical, processing and sustainable prospects, Natural Products and Bioprospecting, 7, 123-145. 